\title{
THE ADVANTAGES OF VELOCITY VECTOR REFERENCING IN PROPORTIONAL NAVIGATION
}

\author{
Pravas R. Mahaptra and Uday S. Shukla \\ Department of Aerospace Engineering \\ Indian Institute of Science \\ Bangalore 560012, India
}

\begin{abstract}
Two generic classes of Proportional Navigation (PN) laws exist depending on whether the control acceleration of the pursuer is referenced relative to the pursuer velocity vector or the target-pursuer line-of-sight (LOS). This paper makes a critical comparison of the two types of laws, covering aspects of their implementation, analysis and trajectory behavior. It is shown that the LOSreferenced PN laws possess serious limitations in terms of implementation and trajectory behavior. These include forward acceleration and braking as a part of the control effort, relative inefficiency in terms of control effort requirements, restrictive launch conditions and singularities in acceleration requirements.
\end{abstract}

\section{INTRODUCTION}

In one form or another, Proportional Navigation (PN) is used very frequently as a guidance law for intercept and rendezvous in aerospace applications. Its greatest advantage in such applications is the relative simplicity of implementation. The PN guidance requires very low levels of information, consisting essentially of the pointing direction from the pursuer to the target and its rate of sweep. The pointing direction, i.e. the target-pursuer line-of-sight (LOS), is readily sensed on board even small and simple platforms using simple and low-cost tracking devices based on microwave, infrared, optical or acoustic trackers. The guidance computer determines a control force proportional to the LOS rotation rate, which is applied to the pursuer through either control surface deflection or the use of control thruster(s).

In modeling of systems based on the PN principle to be able to predict their behavior, serious analytical difficulties are encountered. This is because the differential equations governing the pursuer motion under PN law are highly nonlinear, even when only the kinematics are considered. Two principal approaches have been made in analyzing PN systems. These correspond to two slightly different definitions of the PN law. In one definition the pursuer velocity vector serves as the reference for the application of the control force, resulting in the Pure Proportional Navigation (PPN) and its variants. The other definition utilizes the LOS as a reference, and leads to laws like the True Proportional Navigation (TPN) and its generalizations.

In this paper, a critical comparison is made between the two types of guidance laws. The comparison includes the facility of analysis as well as the behavior of the pursuer under the two guidance laws. In certain respects, the LOS-referenced laws appear to have an analytical advantage over the velocity-vector-referenced laws. However, the comparison made in this study shows that such advantage is indeed limited. On the other hand, LOS-referenced laws lead to highly undesirable trajectory behavior and suffer from serious difficulties in their implementation.

\section{THE TWO CLASSES OF PN LAWS}

PN was initially defined $[1,2]$ as a navigation law in which a pursuing point moves toward a target point in a plane containing the velocity vectors of the two points in such a manner that the direction of the velocity vector (heading) of the pursuer is rotated at a rate proportional to the rotation rate of the line joining the pursuer and the target (i.e. the LOS). Later definitions consider the pursuer lateral acceleration, rather than the heading, to vary in proportion with the LOS rate.

The pursuer can be made to obey the PN law by applying a controlled force to it, which will result in a 


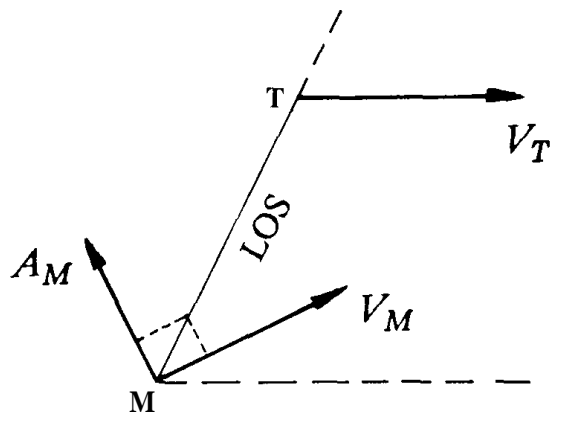

Fig. 1 Schematic showing the direction of application of control force on pursuer in Pure Proportional Navigation (PPN).

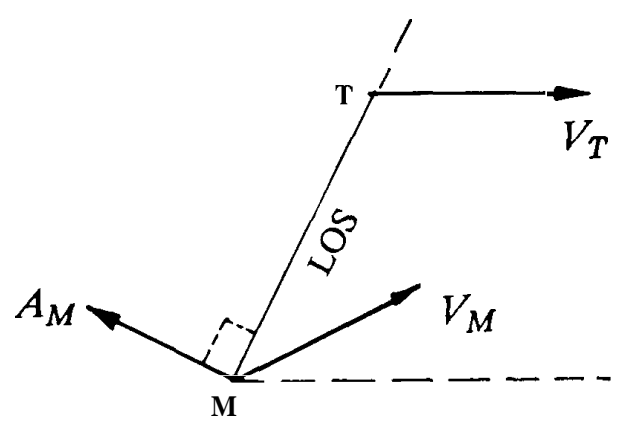

Fig. 2 Schematic showing the direction of application Proportional Navigation (TPN).

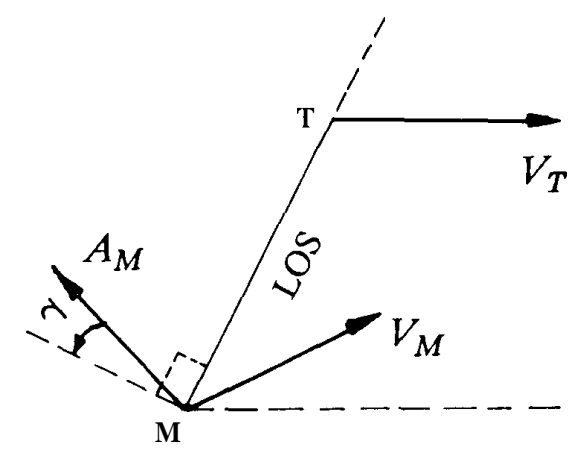

Fig. 3 Schematic showing the direction of application of control force on pursuer in Generalized True Proportional Navigation (GTPN). controlled maneuver. The basic PN principle establishes the magnitude of the control force (and hence maneuver or acceleration) to be proportional to the LOS turn rate. However, depending on the direction of application of this force several slightly different definitions of PN are possible. Three of these are significant $[3,4,5]$ :

1. Pure Proportional Navigation (PPN): The desired lateral acceleration (proportional to the LOS turning rate) is applied normal to the velocity vector of the pursuer. The scheme is shown in Figure 1.

2. True Proportional Navigation (TPN): The control force (and hence acceleration) is applied normal to the instantaneous LOS from the pursuer to the target. The scheme is depicted in Figure 2.

3. Generalized True Proportional Navigation: The control force is not necessarily applied normal to the LOS, but has a fixed angle relative to it. The scheme is shown in Figure 3.

The first of these is a velocity-vector-referenced law, and the other two are referenced with respect to the LOS.

\section{PURE PROPORTIONAL NAVIGATION (PPN)}

The kinematic variables relating to a general planar pursuit against a maneuvering target are shown in Figure 4. The target $T$ and the pursuer $M$ have constant velocities $V_{T}$ and $V_{M}$ and normal accelerations $\boldsymbol{A}$, (assumed constant) and $\boldsymbol{A}$, respectively. The pursuit is described in a coordinate system centered at $T$ and with respect to a reference line parallel to the initial direction of $V_{T}$.

The differential equations of motion are obtained by resolving the target and pursuer velocities along and normal to the LOS and performing certain algebraic operations, as

$\dot{r}=V_{T} \cos (\theta-\beta)-V_{M} \cos (b \theta-c)$

and

$r \theta=-V_{T} \sin (\theta-\beta)+V_{M} \sin (b \theta-c)$

where

$b=1-N$ and $c=\phi_{i}-N \theta_{i}$ 


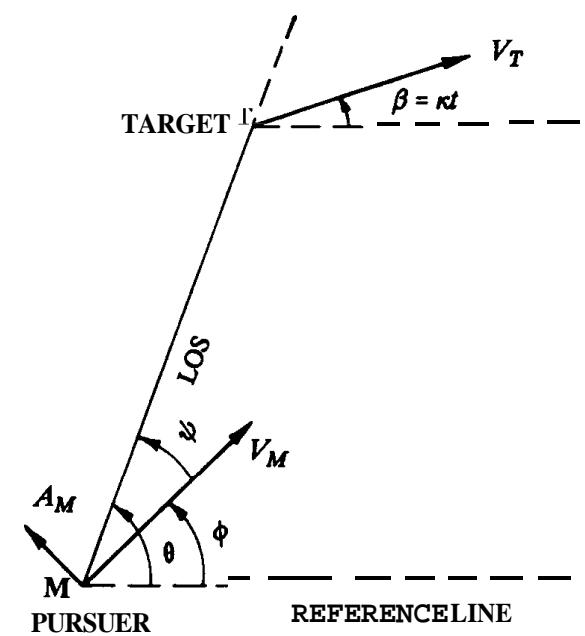
Fig. 4 Pure Proportional Navigation (PPN) geometry
for planar pursuit of a maneuvering target.

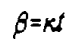

and

$\kappa=A_{T} / V_{T}$

represents the turn rate of the target heading. The subscript $i$ denotes initial values.

The system of equations (1) and (2) describing the motion of the pursuer against a general maneuvering target are highly nonlinear. These have not been solved in closed form for any value of the navigation constant $\boldsymbol{N}$ which is the constant of proportionality between the turn rates of the pursuer heading and the LOS, i.e.

\section{$\dot{\phi}=N \dot{\theta}$}

For the relatively simple case when the target is not maneuvering, $B=0$ and the right hand sides of equations (1) and (2) have no explicit dependence on time, being functions of $\theta$ only. Even for this "simple" case, no general solution has been published, but exact solutions have been obtained [1] for $\mathrm{N}=\mathbf{1}$ and $\mathbf{2}$ only.

The case $\mathrm{N}=1$ reduces PPN to a case of simple pursuit, i.e. pure pursuit when $\boldsymbol{\phi}_{\boldsymbol{i}}=\boldsymbol{\theta}_{\boldsymbol{i}}$ (pursuer initially heading toward the target) and deviated pursuit when the equality is not true. Both of these cases are solvable
[1]. The solution for $\mathrm{N}=\mathbf{2}$ is incomplete in the sense that the radial distance $r$ can be solved in terms of $\boldsymbol{\psi}$ or $\theta$ (Figure 4) $[1,6]$, but an expression for the time $t$ cannot be found in terms of these trajectory variables.

Practical PN systems utilize values significantly larger than $\mathbf{2}$ for the navigation constant $\boldsymbol{N}$ to obtain desirable pursuer trajectory response characteristics. In the absence of solutions for the PPN equations for such values of the constant, even for non-maneuvering targets, linearized solutions (e.g. (2]) have hitherto been used to investigate $\mathrm{PN}$ behavior for realistic engagements and to optimize $\boldsymbol{N}$ with respect to maneuver, time or energy requirements.

The linearized formulations of PPN have been based on the assumption that the engagement geometry stays close to a collision course with a small LOS angle $\theta$ and that the target heading $B$ does not change much from its initial direction $\boldsymbol{B}_{i}$ during the entire engagement. Such assumptions lead to a linear time-varying differential equation of the first order for the LOS rate. The equation is homogeneous for a non-maneuvering target and non-homogeneous for a maneuvering target, and is readily solved [2]. The linear solutions are quite accurate for small values of $\theta, \Delta \phi_{i}$ (initial heading error relative to the collision course) and A, Such conditions effectively describe a geometry close to a tail chase against a low-maneuver target. The solutions become increasingly inaccurate for engagements departing from such conditions, becoming rather unacceptable for large values of these parameters.

The severe limitations of the classical linear solutions in terms of the engagement geometry and maneuver levels have been significantly obviated by the use of a quasilinear approach. By confining attention to terms only up to the first derivative and considering only one iteration, it is possible to obtain explicit analytical solutions for all the trajectory parameters for both nonmaneuvering as well as maneuvering targets $[7,8]$. Such solutions have the merit of providing accurate analytical estimates of the trajectory parameters for engagement conditions that are significantly far from tail-chase and for highly maneuvering targets.

In addition to the linear analysis, further insight has been gained into the behavior of the PPN scheme through qualitative analysis $[9,10,11]$. Such treatment has provided conditions for intercept in relation to initial states and bounds on the pursuer acceleration, but has not provided any solutions, explicit or implicit, for any of 
the motion parameters.

\section{TRUE PROPORTIONAL NAVIGATION (TPN)}

In TPN, the control force is applied normal to the instantaneous LOS. For this situation, the kinematic equations of pursuer motion, considering a nonmaneuvering target, are

$$
\ddot{r} \cdot r \ddot{\theta}^{2}=0
$$

and

$$
r \theta+2 r \theta=-C \theta
$$

where

$c=-x v$,

$V_{R i}$ is the initial relative closing velocity along the LOS and $\lambda$ is a constant. TPN was treated in [5] using a linearized approach and considering a non-maneuvering target; the solutions were similar to those for PPN.

The equations of motion (7) and (8) can be combined and written, after some simplification, as $[3,6]$

$\ddot{r}+(3+2 C) \ddot{r}=0$

which is in terms of $r$ only. This equation has been solved in closed form [3]. The solution is implicit and uses indirect variables which somewhat blur the physical insight into TPN behavior, but such transformation of variable from direct to indirect is necessary for solving the equation.

The univariate form (10) is possible only if the right hand side of (7) is zero, as in the case of nonmaneuvering targets. Such is not the case for a maneuvering target and/or a further generalization of TPN. Thus, the currently available solution of (10) does not appear to be extendable to more general TPN applications than the basic law applied to nonmaneuvering targets only.

Both TPN and PPN reduce to the collision course as the heading error vanishes. Thus, the linearized solutions of both are similar. However, as conditions depart significantly from the collision course, the behavior of the two schemes is remarkably different. In particular, intercept of a non-maneuvering target in the case of PPN is assured, under certain liberal conditions that are nearly always satisfied, for all directions of initial pursuer heading except a precisely defined one [9], whereas TPN assures intercept only when the initial conditions lie within a determined circle, termed the circle of capture.

\section{GENERALIZED TRUE PROPORTIONAL NAVIGATION (GTPN)}

A generalized true proportional navigation (GTPN) has recently been proposed [4] with the aim of minimizing the limited-capture-area drawback of the TPN. In this scheme, the control force/acceleration of the pursuer is not necessarily applied normal to the LOS (as is the case with TPN) but maintains a fixed angle $\gamma$ with respect to the normal to the LOS. TPN is a special case of GTPN when $\gamma=0$.

The GTPN kinematic equations are

$$
\ddot{r}-r \dot{\theta}{ }^{2}=\lambda V_{a} \sin \gamma
$$

and

$r \dot{\theta}+2 \dot{r} \theta=-\lambda V_{R \dot{c}} \cos \gamma$

As discussed above, the method [3] used to solve the TPN equations cannot be generalized to solve the system of equations (11) and (12) representing the GTPN case. A much more involved procedure has been used in [4] to solve the equations using the LOS angle $\theta$ as the independent variable, but the solution is implicit in nature. Although an inequality defining the capture area is obtained, no explicit expressions for either any of the trajectory parameters such as $8, \dot{\theta}$ and $\boldsymbol{A}$, or time $\boldsymbol{t}$ are presented.

GTPN has a larger capture area (boundaries of initial conditions which result in intercept) than TPN under certain conditions [4]. However, for slightly large values of $\gamma$, for which the advantage of larger capture area is claimed, GTPN results in unbounded LOS rate and control force even for normal values of navigation constant such as $\lambda=3$. This highly undesirable trajectory behavior more than offsets the advantage of the slightly higher capture area.

A further generalization of GTPN is the Generalized Guidance Law (GGL) [12] in which the applied control 
force is made proportional to the rate of change of a generalized vector in a two-dimensional space. Depending on the choice of two functions used in the definition of the generalized vector, a family of LOSreferenced guidance laws result, which include the GTPN and the TPN.

GTPN is indeed quite general in terms of its definition, though its solution has been found tractable only for the familiar schemes of TPN, GTPN and Prediction Guidance Law [13], and that too only for nonmaneuvering targets. However, since the LOS has no fixed relationship with the pursuer velocity vector, even the most general LOS-referenced guidance law will not include the PPN in which the control effort is referenced relative to the pursuer heading. Hence the solution methods developed for the LOS-referenced laws cannot be applied to the treatment of the PPN problem.

\section{COMPARATIVE ASPECTS OF THE TWO CLASSES OF LAWS}

Based on the characteristics of the individual schemes discussed in the sections above, it is now possible to make a comparison between them with regard to their implementation, method and nature of the solutions of their equations of motion, and the behavior of their resulting equations of motion. Such a comparison is made in the paragraphs below.

\section{Implementation Aspect: Forward Speed Variation}

In PPN the control force is applied normal to the pursuer velocity vector. Since there is no component of acceleration along the pursuer heading direction, the pursuer forward velocity $V_{M}$ will remain constant throughout the engagement, neglecting external forces. Such is not the case with TPN in which the control force is applied normal to the LOS, having components both along and normal to the pursuer velocity vector. The forward acceleration component (i.e. fhe component along the pursuer heading direction) is $V_{M}=-A_{M} \sin (\theta-\phi)$.

Similar is the case with GTPN, in which the pursuer acceleration is applied at a fixed angle $\boldsymbol{\gamma}$ with respect to the normal to the LOS. Thus, in this case too, the control force will in general have components both along and normal to the pursuer .velocity vector; the forward acceleration component is $V_{M}=-A_{M} \cos \gamma \sin (\theta-\phi)$.

The forward acceleration $V_{M}$ in the case of TPN is shown graphically in Figure 5, plotted against the normalized range $r / r_{i}$. These plots have been obtained from the basic differential equations of TPN motion by numerical integration. In contrast, a plot of forward acceleration for PPN would show a uniformly zero value throughout the engagement. The graphs of Figure 5 confirm the fact that the TPN scheme requires considerable acceleration and/or deceleration along the flight direction of the pursuer. Similar would be the case with other LOS-referenced PN schemes. The effect is vary pronounced for large departures of the engagement geometry from the collision course. Since most pursuing vehicles use aerodynamic/hydrodynamic control surfaces, such forward acceleration and deceleration is impossible to apply in a controlled manner, making the implementation of LOS-referenced laws extremely difficult. If reaction-type control is employed, as is normal for extra-atmospheric maneuvers, controlled forward acceleration and deceleration is possible, but is both cumbersome and wasteful. This latter aspect is discussed in the section below.

Continued forward acceleration/deceleration would cause the forward velocity of the pursuer to vary during the engagement in the case of LOS-referenced laws. The normalized $V_{*}$ plots for TPN in Figure 4 illustrate this. While similar plots for PPN would have a constant value of unity throughout the engagement, the speed history of TPN shows pronounced variations. Such large speed changes would result in altered aerodynamic characteristics and control system behavior, leading to difficult autopilotage.

\section{Control Efficiency}

Table I shows the kinematic control efficiency of the two types of PN .. the velocity-vector-referenced and the LOS-referenced -- using the PPN and the TPN as respective examples. The control efficiency is indicated by the integral of the magnitude of the lateral acceleration [5]. It is clearly apparent from Table I that

Table I Integrated control acceleration AV for True Proportional Navigation (TPN) and Pure Proportional Navigation (PPN).

\begin{tabular}{|c|c|c|c|c|c|c|c|}
\hline \multirow[t]{2}{*}{$\theta$} & \multirow[t]{2}{*}{$\Delta \phi_{i}$} & \multicolumn{2}{|c|}{$N=2$} & \multicolumn{2}{|c|}{$\mathbf{N}=\mathbf{3}$} & \multicolumn{2}{|c|}{$N=4$} \\
\hline & & TPN & PPN & TPN & PPN & TPN & PPN \\
\hline $\begin{array}{l}15 \\
30 \\
60\end{array}$ & $\begin{array}{r}5 \\
15 \\
40\end{array}$ & $\begin{array}{r}15.47 \\
43.76 \\
123.6\end{array}$ & $\begin{array}{l}15.47 \\
42.88 \\
96.82\end{array}$ & $\begin{array}{l}11.30 \\
33.15\end{array}$ & $\begin{array}{l}11.29 \\
32.80\end{array}$ & $\begin{array}{r}9.84 \\
29.07\end{array}$ & $\begin{array}{r}9.80 \\
28.72 \\
71.68\end{array}$ \\
\hline
\end{tabular}



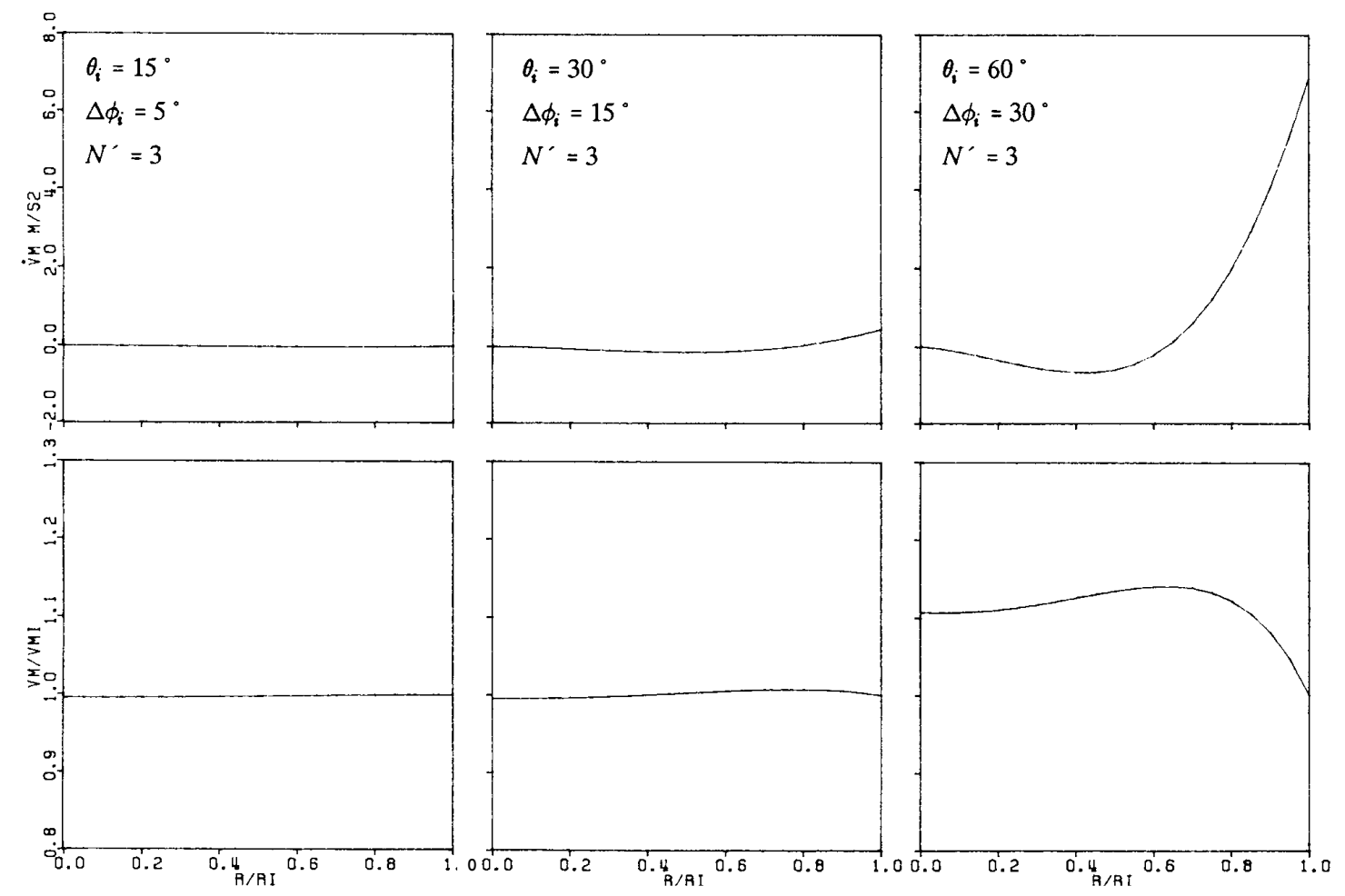

Fig. 5 Variation of forward velocity (normalized) and forward acceleration of the pursuer as a function of normalized range-togo for True Proportional Navigation (TPN). For Pure Proportional Navigation (PPN), the normalized forward velocity is unity and the forward acceleration is zero at all ranges.

PPN consistently requires a lower control effort than TPN for a wide range of geometries and values of the navigation constant. Since PPN and TPN approach each other for near-tail-chase geometries, their control efficiencies are similar for such engagements. However, at larger departures from tail-chase and collision-course conditions, the disadvantage of TPN is very significant. The relative inefficiency of the TPN is caused directly by the energy lost in the forward acceleration and/or deceleration of the pursuer as a part of the control effort.

\section{Analytical Aspects}

It was mentioned earlier in connection with the discussion on TPN that the current solutions for the TPN equations are possible only for the special form of the equations that arise when the engagement is against a non-maneuvering target. Even for this restricted case, the solutions are indirect and implicit. The solutions to the GTPN case have been obtained with respect to the LOS angle $\theta$. These solutions are also implicit. The solution helps obtain an inequality representing the capture region, i.e. the boundaries of initial conditions which lead to intercept, but do not yield explicit expressions for any of the trajectory parameters. The "general solution" of the GTPN can be evaluated only for relatively simple TPN variants, and only when the target is not maneuvering.

Even such limited solvability has not yet been possible for the PPN equations in an exact sense. The exact solutions for PPN are much more restricted, concerning non-maneuvering targets and only two discrete (and practically unimportant) values of the navigation constant.

\section{Trajectory Behavior: Capture Area}

When the pursuit is started with the pursuer and 
target closing in at a significant rate and the LOS rotating relatively slowly, which is a condition frequently found in practice, GTPN can provide a larger capture area than TPN [6]. PPN, however, requires much more liberal initial conditions for intercept: impact is always possible except for one highly improbable launch direction if the navigation constant exceeds 2 which is invariably the case. Thus, TPN and even GTPN are much more restrictive than PPN in terms of initial conditions for intercept.

\section{Trajectory Behavior: Singularities}

The pursuer lateral acceleration in the case of PPN is generally well-behaved, being a uniformly decreasing function of time for the major part of commonly used pursuer-to-target speed ratios [6]. TPN imposes tighter conditions on the initial LOS rate for the lateral acceleration to remain finite during the engagement [3]. The behavior of GTPN is further degraded with regard to pursuer lateral acceleration requirements. Here, even for many normal values of the navigation constant, the LOS rate (to which the control force is proportional) becomes unbounded even for small values of $\gamma$, i.e. even if GTPN departs only marginally from TPN [4]. For larger $\gamma$ values, for which GTPN is supposed to improve capture area restrictions with respect to PPN, the difficulties relating to LOS rate singularities persist.

\section{Trajectory Behavior: Robustness}

It follows from the discussions of the earlier paragraphs that the desirable trajectory behavior (and even the occurrence of intercept) of LOS-referenced laws such as TPN, GTPN and their generalizations is strongly conditioned upon the finer aspects of the initial conditions of engagement. To that extent, these laws lack robustness, since such fine initial conditions may not be possible to guarantee under all engagement scenarios.

\section{Summary}

In summary, the LOS-based PN laws such as TPN, GTPN and GLL are less practical than velocity-vectorreferenced laws such as PPN due to the following reasons:

1. Requirement of forward acceleration and braking, which is not possible with aerodynamic controls and inefficient with the use of thrusters.

2. Inefficiency of control effort
3. Relatively strong restrictions on initial conditions to ensure intercept

4. Unbounded lateral acceleration, especially for GTPN

5. Lack of robustness

\section{CONCLUSIONS}

In this paper, two broad classes of proportional navigation laws have been compared with regard to their implementation, analysis and behavior. The first consists of laws such as the PPN which are referenced relative to the pursuer velocity vector and the second consists of LOS-referenced laws such as TPN, GTPN and GGL. It has been shown that the latter class has serious drawbacks in terms of implementation and trajectory behavior which more than offset the limited advantage in analytical treatment exploited hitherto in literature. Among the major drawbacks are forward acceleration and braking requirements which are difficult to achieve, relative inefficiency in the utilization of control efforts, restrictions on initial conditions for intercept, lack of robustness and the possibility of unbounded acceleration.

From a practical point of view, PPN is a superior guidance law than TPN and its generalizations. The only real utility of the LOS-referenced laws appears to be to serve as approximations to analyze the more practical and efficient PPN law and its variants.

\section{REFERENCES}

[1] Locke, A. S.: Guidance. D. Van Nostrand Co., Princeton, 1956.

[2] Jerger, J. J.: Systems Preliminary Design. D. Van Nostrand Co., Princeton, 1960.

[3] Guelman, M., "The closed form solution of true proportional navigation," IEEE Trans. on Aerospace and Electronic System, Vol. AES-7, pp. 472-482, 1976.

[4] Yang, C-D., F-B. Yeh and J-H. Chen, 'The closed form solution of generalized proportional navigation," Journal of Guidance, Control and Dynamics, Vol. 10, pp. 216-218, 1987. 
[5] Murtaugh, S. A. and H. E. Criel, "Fundamentals of proportional navigation," IEEE Spectrum, Vol. 3, pp. 75 85, 1966.

[6] Shukla, U. S. and P. R. Mahapatra, "The proportional navigation dilemma -- Pure or true ?," To appear in IEEE Trans. on Aerospace arid Electronic Systems, March 1990.

[7] Shukla, U. S. and P. R. Mahapatra, "A generalized linear solution of proportional navigation," IEEE Trans. on Aerospace and Electronic Systems, Vol. AES-24, pp. 231-238, 1988.

[8] Shukla, U. S. and P. R. Mahapatra, "Accurate solution of proportional navigation for maneuvering targets," IEEE Trans. on Aerospace and Electronic Systems, Vol. AES-25, pp. 81-89, 1989.
[9] Guelman, M., "A qualitative study of proportional navigation," IEEE Traits. on Aerospace and Electronic Systems, Vol. AES-7, pp. 337-343, 1971.

[10] Guelman, M., "Proportional Navigation with a maneuvering target," IEEE Trans. on Aerospace and Electronic Systems, Vol. AES-8, pp. 364-371, 1972.

[11] Guelman, M., "Missile acceleration in proportional navigation," IEEE Trans. on Aerospace and Electronic Systems, Vol. AES-9, pp. 462-463, 1973.

[12] Yang, C-D., F-B. Hsiao and F-B. Yeh, "Generalized guidance law for homing missiles," IEEE Tram. on Aerospace and Electronic Systems, Vol. AES-25, pp. 197. 212, 1989.

[13] Kim, Y. S., H. S. Cho and Z. Bien, "A new guidance law for homing missiles," Joumal of Guidance, Control arid Dynamics, Vol. 8, pp. 402-404, 1985. 